\title{
ON THE N-TH ORDER RICCATI EQUATION OF THE SECOND KIND
}

$\mathrm{BY}$

T. IWIŃSKI (WARSAW)

§ 1. In [1] a theory of a certain class of the $n$-th ordor non-linear differential equations was given: they are called generalized Riccati equations (oì, in short, $R$-equations) and are closely connected with linear equations of the $(n+1)$-st order $L_{n+1}[y]=0$. The definition of these equations required the assumption of multiple differentiability of the coefficients of the corresponding linear equation. In the present paper, using weaker assumptions, namely assuming only continuity of the coefficients, shall define another kind of non-linear equations of the $n$-th order, with properties related to those of $R$-equations. To distinguish them, we shall eall them Ricati equations of the second kind, or $\hat{R}$-equations.

§ 2. Let us consider a function $u(x)$ defined in the interval $(a, b)$ and belonging to the class $C^{k}$. Under this assumptions, to every integer $n \leqslant k$, we shall assign a non-linear differential operator $I^{n}$ defined on function $u(x)$ by the following recursive relation (see $[1], p .6$ ):

$$
I^{0}[u]=u, \quad I^{n}[u]=\frac{d}{d x} I^{n-1}[u]+u I^{n-1}[u]
$$

Definition. The equation.

$$
\hat{R}_{n}[u] \equiv a_{n+1,0}+\sum_{i=1}^{n+1} a_{n+1, i} I^{i-1}[-u]=0
$$

will be called $n$-th order Riccati equation of the second kind, or, briefly $\hat{R}_{n}$-equation. We shall assume that the coefficients $a_{n+1, i}(i=0,1, \ldots$ $\ldots, n+1)$ are defined and continuous in $(a, b)$.

The simplest equation. $\hat{R}_{1}$ is of the form

$$
a_{22} u^{\prime}=a_{22} u^{2}-a_{21} u+a_{20},
$$

thus, it is the classical equation of Riccati. 
83. Having defined by (2) the $\hat{R}_{n}$-equation, we shall consider the following linear differential equation of the $(n+1)$-st order, whose coefficients will be the functions $a_{n+1, i}(i=0,1, \ldots, n+1)$ which appear in (2):

$$
L_{n+1}[y] \equiv a_{n+1,0} y+\sum_{i=1}^{n+1} a_{n+1, i} y^{(i)}=0 .
$$

The relations between the equations (3) and (2) are given in the following theorem:

THeorem 1. Every $\hat{R}_{n}$-equation of the $n$-th order can be reduced to the linear equation of the $(n+1)$-st order by changing variables $u=-y^{\prime} / y$.

Every homogeneous linear equation of the $(n+1)$-st order can be reduced

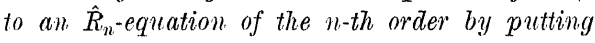

$$
y=\exp \left[-\int u d x\right] \text {. }
$$

Proof. It can be shown by induction, that from the definition (1) of the operator $I^{n}$ follow the formulas:

$$
\begin{gathered}
I^{n}[-u]=y^{(n+1)} / y \quad\left(u=-y^{\prime} / y\right) \\
y^{(n)}=e^{-U} I^{n-1}[-u],
\end{gathered}
$$

where $U=\int u(x) d x$ (we shall keep this notation in further considerations).

It follows at once that

$$
\begin{aligned}
y \hat{R}_{n}\left[-\frac{y^{\prime}}{y}\right] & =L_{n+1}[y], \\
L_{n+1}\left[e^{-U}\right] & =e^{-U} \hat{R}_{n}[u] .
\end{aligned}
$$

Formulas (7) and (8) imply both assertions of our theorem. Subsequently we give some properties $(\mathrm{I}-\mathrm{V})$ of $\hat{R}_{n}$-equations derived from theorem 1.

I. The solution of the nom-linear $\hat{R}_{n}$-equation can be reduled to the solution of linear equation. (3) (and conversely).

This property is well-known in the case of the classical Riceati equation.

If the function

$$
y=\sum_{i=1}^{n+1} G_{i} y_{i} \quad\left(C_{i}=\text { const }\right)
$$

is a solution of the linear homogeneous equation $L_{n+1}[y]=0$ of the $(n+1)$-st order, then the function

$$
u=-\frac{\sum_{i=1}^{n+1} C_{i} y_{i}^{\prime}}{\sum_{i=1}^{n+1} C_{i} y_{i}}
$$

is the general solution of the eorresponding $\hat{R}_{n}$-equation.

II. Suppose that we are given a system of $n+1$ functions

$$
u_{1}, u_{2}, \ldots, u_{n+1}
$$

from $C^{n}(a, b)$. We shall consider the following functional determinant:

$$
\hat{T}\left(u_{1}, \ldots, u_{n+1}\right)=\left|\begin{array}{llll}
1 & 1 & \ldots & 1 \\
I^{0}\left[u_{1}\right] & I^{0}\left[u_{2}\right] & \ldots & I^{0}\left[u_{n+1}\right] \\
I^{1}\left[u_{1}\right] & I^{1}\left[u_{2}\right] & \ldots & I^{1}\left[u_{n+1}\right] \\
\ldots \ldots \ldots \ldots & \ldots & \ldots & \ldots \ldots \\
I^{n-1}\left[u_{1}\right] & I^{n-1}\left[u_{2}\right] & \ldots & I^{n-1}\left[u_{n+1}\right]
\end{array}\right| .
$$

If the functions $u_{i}(i=1,2, \ldots, n+1)$ are particular solutions of an $\hat{R}_{n}$-equation of the $n$-th order, and if the functions (11) satisfy in $(a, b)$ the condition

$$
\hat{T}\left(-u_{1},-u_{2}, \ldots,-u_{n+1}\right) \neq 0,
$$

then the general solution of the corresponding linear equation of the $(n+1)$-st order is the function.

$$
y=\sum_{i=1}^{n+1} C_{i} e^{-t_{i}}
$$

In fact, it follows from (8) that each of the functions $y_{i}=\exp \left(-U_{i}\right)$ is a particular solution of equation (3). It remains to show that these functions are linearly independent in $(a, b)$, which, however, follows at onee from the assumption that they satisfy condition (13). In fact, if functions (1.1) satisfy condition (13), then from the easily verified relation

$$
W\left(e^{-U_{1}}, e^{-\tau_{2}}, \ldots, e^{-V_{n+1}}\right)=\exp \left[-\sum_{i=1}^{n+1} U_{i}\right] \hat{T}\left(-u_{1}, u_{2}, \ldots,-u_{n+1}\right),
$$

where $W$ denotes the Vronsky determinant, follows the linear independence of the functions $y_{i}=\exp \left(-U_{i}\right)$, which was to be shown. 
III. If the function $u\left(x, C_{1}, \ldots, C_{n}\right)$ is a general solution of the equation $\hat{R}_{n}[u]=0$, then the corresponding linear equation $L_{n+1}[y]=0$ has the solution

$$
y=C_{n+1} \cdot e^{-U\left(x, C_{1}, \ldots, C_{n}\right)} \quad\left(C_{i}=\text { const }\right)
$$

In fact, it follows from the formula $u=-y^{\prime} \mid y$ that $y$ satisfies the differential equation of the first order:

$$
y^{\prime}+u\left(x, C_{1}, C_{2}, \ldots, C_{n}\right) y=0
$$

hence we have (16).

IV. An $\hat{R}_{n}$-equation with coefficients defined and continuous in the interval $(a, b)$ has a solution in the domain of real functions in the whote interval $(a, b)$, except at most a countable number of points. In the domain of complex functions of real variable, an $\hat{R}_{n}$-equation has, under the same assumptions, a solution in the whole interval $(a, b)$.

In fact, to every equation (2) we can assign a linear equation (3). Thus the property IV follows from formula (10) and from the corresponding properties of the solution of linear equation (3), whose coefficients are continuous in $(a, b)$.

To show the second part it is sufficient to use the following

Theorem of G. Mammana (see [2], theorem 1). For the most general linear differential equation of the $n$-th order there exists an arbitrary number of pairs of particular solutions which do not vanish simultaneously at any point.

In this theorem the usual continuity in the whole interval under consideration, is assumed.

$\mathrm{V}$. If we know $n$ solutions $u_{n i}$ of the equation, $\hat{R}_{n}[u]=0$ such that the functions $-u_{n i}(i=1,2, \ldots, n)$ form a system of essentially different functions, $i . e$. they satisfy the condition $\hat{T}\left(-u_{n 1},-u_{n, 2}, \ldots,-u_{n, n}\right) \neq 0$. then the general solution of this equation can be obtained by quadratures,

Indeed, under the assumptions of this theorem we know a system of linearly independent solutions of the form $y=\exp \left(-U_{n i}\right)$ of the linear equation $L_{n+1}[y]=0$, which corresponds to the given equation $\hat{R}_{n}[u]=0$ (property II, formula (1.5)). But, as we know, in this case the last linearly independent solution $y_{n+1}$ can be found by quadratures; thus the general solution of an $\hat{R}_{n}$-equation is defined by $(10)$, which was to be shown.

We can formulate analogous properties in the case when we know $n+1$ particular solutions $u_{n i}(i=1,2, \ldots, n+1)$ of an $\hat{R}_{n}$-equation.

§4. We can obtain further properties of $\hat{R}_{\eta}$-equations using the considerations concerning the drcomposition of the differential expression $L_{n+1}[y]$ into operator factors (this idea is due to Floquet (see [3], p. 267).

It has been shown in [1] on Riceati equations of the first kind, that one can connect with these equations the decomposition of differential expression $L_{n+1}[y]$ into operator factors of the form

$$
L_{n+1}[y]=\left(\frac{d}{d x}+a_{n i}\right) L_{n}[y] .
$$

In the case of the Riceati equation of the second kind, i. e., in the case of an $\hat{R}_{n}$-equation (2), one can connect with these equations a decomposition of linear expression into the following operator factors:

where

$$
L_{n+1}[y]=L_{n}\left[\frac{d y}{d x}+u_{n} y\right]
$$

$$
L_{n}=a_{n 0}+\sum_{i=1}^{n} a_{n i} \frac{d^{i}}{d x^{i}} .
$$

We assume that the function $u_{n}(x)$ belongs to the class $C^{n}(a, b)$, and that the functions $a_{n i}(i=0,1, \ldots, n)$ are continuous. In order to obtain the decomposition (18) we have to define the functions $a_{n i}(i=0$, $1, \ldots, u)$ and $u_{n}$ in such a way that the relation (18) may hold. If such functions exist, we shall call them coefficients of the decomposition, and equation (3) will be called decomposable in the interval $(a, b)$. The coefficient $u_{n}$ will be called distinguished coefficient of the decomposition.

THEOREM 2. The function $u_{n}$ is a distinguished coefficient of the decomposition. (18) if and only if this function is a particular solution of the $\hat{R}_{n}$-equation (2) corresponding to the linear differential equation (3).

Proof. The first part of the theorem is obvious: if the linear equation (3) is decomposable, and $u_{n}$ is a distinguished coefficient of the decomposition, then the function $y=\exp \left(-U_{n}\right)$ satisfies equation (3.), and hence, the function $\psi_{n}$ is a solution of the $\hat{R}_{n}$-equation (2).

Thus, it remains to show that each solution $u_{n}$ of equation (2) determines a certain system of coefficients $a_{n i}(i=0,1, \ldots, n)$, which, together with $u_{n}$, form a system of functions satisfying (18) in the interval under consideration.

To do that we shall-derive a system of equations for the coefficients of the decomposition of the linear differential equation into operator factors.

Colloquium Mathematicum $\mathrm{x}$. 
We shall use the following auxiliary formula (which oan be easily proved by induction):

$$
L_{n}\left[u_{n} y\right]=\sum_{i=0}^{n} B_{n i} y^{(n-i)}
$$

where

$$
B_{n i}=\sum_{k=0}^{i} a_{n, n+k-i}\left(\begin{array}{c}
n+k-i \\
k
\end{array}\right) u_{n}^{(k)} \quad(i=1,2, \ldots, n) .
$$

The symbols $u_{n}(x)$ and $y(x)$ denote functions from the class $C^{n}(a, b)$, and $\left(\begin{array}{l}0 \\ 0\end{array}\right)=1$.

Let us re-write identity (18) in an equivalent form

$$
L_{n}\left[u_{n} y\right]+L_{n}\left[y^{\prime}\right] \equiv L_{n+1}[y]
$$

Using formula (20) we find

$$
\sum_{i=0}^{n} B_{n i} y^{(n-i)}+\sum_{i=0}^{n} a_{n, n-i} y^{(n+1-i)}-\sum_{i=0}^{n+1} a_{n+1, n+1-i} y^{(n+1-i)}=0
$$

$$
\begin{aligned}
& \left(a_{n, n}-a_{n+1, n+1}\right) y^{(n+1)}+\sum_{i=1}^{n}\left[B_{n, i-1}+a_{n, n-i}-a_{n+1} n+1-i\right] y^{(n+1-i)}+ \\
& +\left(B_{n, n}-a_{n+1,0}\right) y=0 .
\end{aligned}
$$

For this identity to be satisfied it is necessary and sufficient that the coefficients of the decomposition satisfy the following system of equations:

$$
B_{n, i-1}+a_{n, n-i}-a_{n+1, n+1-i}=0 \quad(i=1,2, \ldots, n)
$$

$$
B_{n, n}-a_{n+1,0}=0,
$$

where $a_{n+1, n+1}=a_{n, n}$.

Thus, the problem of decomposition of linear expression $L_{n+1}[y]$ is reduced to the solution of system (21), consisting of $n+1$ equations, where the unknowns are the decomposition coefficients $a_{n i}(i=0,1, \ldots$, $n-1)$ and $u_{n}$.

As for this problem, it may be reduced to the solution of only one equation. We obtain this equation by eliminating the functions $a_{n i}$ $(i=0,1, \ldots, n-1)$ from system (21), leaving only the unknown $u_{n}$.
Since all unknowns $a_{n i}$ appear linearly, the result of elimination will have the form of the following equation of $(n+1)$-st order:

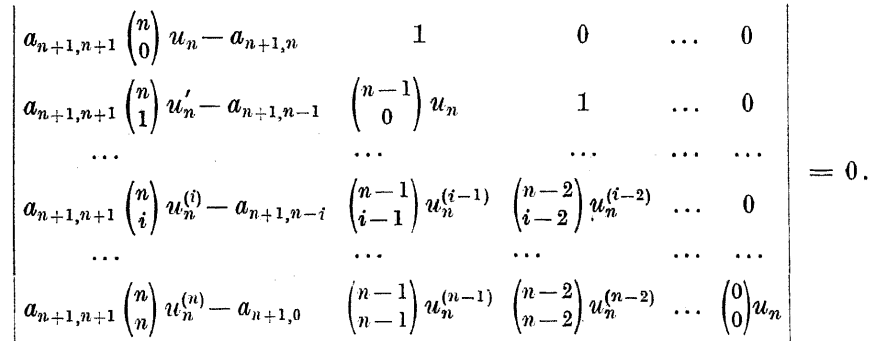

We shall show that equation (22) is an $\hat{R}_{n}$-equation (2), corresponding to equation (3).

To show this let us consider the following functional determinant:

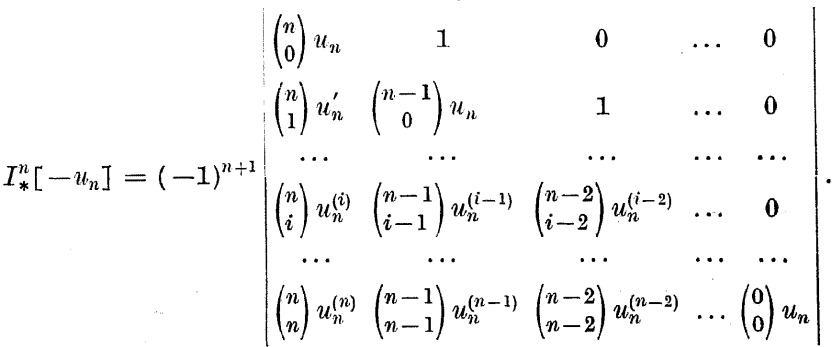

Let us remark that this determinant may be expanded in the following way:

$$
I_{*}^{n}\left[-u_{n}\right]=-\sum_{i=0}^{n-1}\left(\begin{array}{c}
n \\
i
\end{array}\right) u_{n}^{(i)} I_{*}^{n-i-1}\left[-u_{n}\right]-\left(\begin{array}{l}
n \\
n
\end{array}\right) u_{n}^{(n)}
$$

On the other hand, the differential expression (3) can be represented in an analogous way:

$$
I^{n}\left[-u_{n}\right]=-\sum_{i=0}^{n-1}\left(\begin{array}{c}
n \\
i
\end{array}\right) u_{n}^{(i)} I^{n-i-1}\left[-u_{n}\right]-\left(\begin{array}{l}
n \\
n
\end{array}\right) u_{n}^{(n)} .
$$

The proof goes easily by induction, using formula (1) 

identical,

Conclusron. The differential equations $I_{*}^{n}\left[-u_{n}\right]$ and $I^{n}\left[-u_{n}\right]$ are

$$
I_{*}^{n}\left[-u_{n}\right]=I^{n}\left[-u_{n}\right]
$$

for every $u_{n} \in C^{n}(a, b)$.

For the proof we use induction and formulas (24) and (25).

Expanding the determinant (22) with respect to the first column and using (26); we reduce equation $(22)$ to the form (2), which was to be shown.

Since the $\hat{R}_{n}$-equation is a result of eliminating the unknowns from system (21), this system is equivalent to the system formed from (21) by dropping one equation (for instance the last one) and adding the $\hat{R}_{n}$-equation:

$$
\left\{\begin{array}{l}
\hat{R}_{n}[u]=0, \\
B_{n, i-1}+a_{n, n-1}-a_{n+1, n+1-i}=0 \quad(i=1,2, \ldots, n) .
\end{array}\right.
$$

Reduction of system (21) to the form (27) completes the proof. Indeed, to perform the effective decomposition, it is sufficient to take any one of the solutions of equation $\hat{R}_{n}[u]=0$, and then determine $a_{n, n-1}$ from the first equation, $a_{n, n-2}$ from the second equation, and so on, determining in such a way all the coefficients of the decomposition.

By $\S 3$, property IV, and formulas (27), the decomposition of the expression $L_{n+1}[y]$ into factors is, under the assumption of continuity of coefficients $a_{n+1, i}$ in the interval $(a, b)$ assured in the whole interval $(a, b)$ in the domain of complex functions of a real variable.

Now we shall show some consequences of theorem 2 .

I. If we know any particular solution of the $\hat{R}_{n}$-equation (2) corresponing to the non-homogeneous linear equation

$$
L_{n+1}[y]=b_{n+1},
$$

then the integration of this linear equation can be reduced to the integration of a system consisting of two linear equations, of the n-th and of the first order:

$$
\left\{\begin{array}{l}
a_{n n} v^{(n)}+a_{n, n-1} v^{(n-1)}+\ldots+a_{n, 0} v=b_{n+1}, \\
y^{\prime}+u_{n} y=v,
\end{array}\right.
$$

where the coefficients $a_{n i}(i=0,1, \ldots, n)$ are defined by recursive formulas (21). We assume that the function $b_{n+1}$ is continuous in $(a, b)$

This result may be of practical use in cases when we cannot find any particular solution of the linear equation and we have one integral of $\hat{R}_{n}$-equation (2).
In the case when we know a particular solution of the linear equation, our theorem becomes a version of the theorem on the lowering of the order of equation when we know one of its particular solutions (which follows from the formula $u_{n}=-y^{\prime}(y)$. From the point of view of applications, the advantage of such formulation of the theorem on lowering the order of equation lies in the fact of explicit formulas (21) for the coefficients of the linear equation of the lower order.

II. It follows from the assumptions concerning the coefficients of equation (3) and from formulas (21) that the coefficients of the system (29) are continuous in the interval $(a, b)$. Hence, we may apply the procedure formulated above to the linear equation (29), replacing it by the equivalent system of two equations, one of the $(n-1)$-st order, and the other of the first order.

Repeating the above reasonings we get the following conclusion:

For every linear equation (3) of the $(n+1)$-st order with continuous coefficients, there exists an equivalent system of linear equations of the first order; each of these equations contains only one unknown:

$$
\left\{\begin{array}{l}
v_{1}^{\prime}+a_{10} v_{1}=b_{n+1} \\
\ldots \ldots \ldots \\
v_{i}^{\prime}+u_{i-1} v_{i}=v_{i-1} \\
\ldots \ldots \ldots \\
y^{\prime}+u_{n} y=v_{n} .
\end{array}(i=1,2, \ldots, u)\right.
$$

The functions $u_{i}(i=n, n-1, \ldots, 1)$ are particular solutions of equations $\hat{R}_{i}\left[u_{i}\right]=0$ corresponding to equation (3) and next linear equations, respectively, obtained successively by the process of lowering the order of equation by decomposition into operator factors.

The function $a_{10}$ is defined by the formula

$$
a_{10}=a_{n+1, n}-a_{n+1, n-1} \sum_{i=1}^{n} u_{i}
$$

Solving successively the non-homogeneous linear equations (30) we come to the conclusion that there exist particular solutions of linear homogeneous differential equation (with continuous coefficients) of the form

(32) $\quad y_{i}=e^{-U_{n}} \int e^{U_{n}-U_{n-1}} \int \ldots \int e^{U_{n+2-i}-U_{n+1-i}} d x^{i-1}$

$$
\left(i=1,2, \ldots, n+1 ; U_{0}=A_{10}\right),
$$


and the following particular solution of the corresponding non-homogeneous equation (28):

$$
Y_{n+1}=e^{-U_{n}} \int e^{U_{n}-U_{n-1}} \int \ldots \int e^{U_{1}-A_{10}} \int \dot{b}_{n+1} e^{A_{10}} d x^{n+1},
$$

where the capital letters denote the indefinite integrals.

III. Formulas (32) and (33) constitute a convenient tool for proving some theorems in the theory of equations (in spite of the fact that we do not know, in general, the functions $u_{i}(i=1,2, \ldots, n)$; their existence, as we have shown, is assured). We shall illustrate it by a simple example. If we consider a linear equation with constant coefficients and the Euler's equation, then the formulas (32) and (33) lead directly to the well-known formulas for the solutions of these equations, since in this ease, as can be easily seen, $\hat{R}_{u}$-equation (2) becomes an algebraic (characteristic) equation, whose roots determine in a certain way all numbers $u_{i}$.

The methodological simplification here consists in avoiding the separate treatment of the case of multiple roots, and the homogeneous and non-homogeneous equation. The method of variation of constants becomes here unnecessary (see also [1], p. 25).

The idea of reducing linear equations to the non-linear $\hat{R}_{u}$-equations presented in this paper has also certain advantages from the point of view of methods of solving equations. The example given above is not very convincing, since it concerns the equations whose complete solution is known; hence it gives only a new method, without leading to new results. One can, however, show, that there exists a certain class of linear equations with more general functional coefficients, for which the theory of $\hat{R}$-equations presented here does actually lead to the solution, while other methods of solving fail. This problem, however, requires a separate treatment.

\section{REFERENCES}

[1] T. Iwiński, The generalized equations of Riccati and their applications to the theory of linear differential equations, Rozprawy Matematyczno XXIII, Warszawa 1961 .

[2] G. Mammana, Decomposizione delle espressioni differenziali lineari omogenee in prodotti di fattori simbolici e applicazione relativa allo studio delle equazion differenziali lineari, Mathematische Zeitschrift 33 (1931), p. 186.231,

[3] E. Vessiot, Gewöhnliche Differentialgleichungen; elementare Integrationsmethoden, Encyklopädie der Mathematischen Wissenschaften II. I. I, Leipzig $1899-1916$, p. $230-293$

Regu par la Rédaction le \%.10.1961

$\begin{array}{lllllllll}\mathrm{P} & \mathrm{R} & \mathrm{O} & \mathrm{B} & \mathrm{L} & \mathrm{E} & \mathrm{M} & \mathrm{E} & \mathrm{S}\end{array}$

P 212, R 1. Les solutions affirmatives partielles ont été trouvées par Obláth $\left({ }^{1}\right)$, Rosati $\left(^{(2)}\right.$ et Kiss $\left({ }^{3}\right)$.

V. 1, p. 120 .

(1) R. Obláth, Sur l'équation diophantine $\frac{4}{n}=\frac{1}{x_{1}}+\frac{1}{x_{2}}+\frac{1}{x_{3}}$, Mathesis 59 (1950), p. 308-316

(2) L. A. Rosati, Sull'equazione diofantea $\frac{4}{n}=\frac{1}{x_{1}}+\frac{1}{x_{2}}+\frac{1}{x_{3}}$, Bolletino della Unione Matematica Italiana (3), 9 (1954), p. 59-63.

$\left({ }^{3}\right) \mathrm{E}$. Kiss, Quelques remarques sur une équation diophantienne, Studii și cercetări de matematică (Cluj) 10 (1959), p. 59 - 62 (en roumain avec un résumé français).

P 235, R 3. M. Fréchet, l'auteur du problème et de la solution, nous signale d'autres de ses publications parues sur le même sujet $\left({ }^{4}\right)$.

VI, p. 36 ; VIII, p. 289 ; IX, p. 163.

(4) M. Fréchet, Sur une nouvelle définition des semi-espaces de Banach, Comptes rendus des séances de l'Académie des Sciences de Paris 251 (1960), p. 2629 et 2630 La différentielle sur deux semi-espaces de Banach, ibidem 252 (1961), p. 481 et 482; L'espace des courbes est-il un espace de Banach?, Journal de Mathématiques 140 (1961), 11. 197-204. L'espace dont chaque élément est une courbe n'est qu'un semi-espace do Buren, Annales de l'beole Normale Sup rentielle sur un semi-espace de Banach, Bulletin des Sciences Mathématiques 85 (1961) 1). $34-38$.

P 289, R 1. La réponse est négative $\left(^{5}\right)$.

VII, p. 109 et 110

(5) A. Lelek, On weakly ohainable continua, Fundamenta Mathematicae 51 (1962), p. $271-282$ 